PROCEEDINGS OF THE AMERICAN MATHEMATICAL SOCIETY

Volume 124, Number 11, November 1996

\title{
TATE COHOMOLOGY LOWERS CHROMATIC BOUSFIELD CLASSES
}

\author{
MARK HOVEY AND HAL SADOFSKY
}

(Communicated by Tom Goodwillie)

\begin{abstract}
Let $G$ be a finite group. We use recent results of J. P. C. Greenlees and H. Sadofsky to show that the Tate homology of $E(n)$ local spectra with respect to $G$ produces $E(n-1)$ local spectra. We also show that the Bousfield class of the Tate homology of $L_{n} X$ (for $X$ finite) is the same as that of $L_{n-1} X$.

To be precise, recall that Tate homology is a functor from $G$-spectra to $G$-spectra. To produce a functor $P_{G}$ from spectra to spectra, we look at a spectrum as a naive $G$-spectrum on which $G$ acts trivially, apply Tate homology, and take $G$-fixed points. This composite is the functor we shall actually study, and we'll prove that $\left\langle P_{G}\left(L_{n} X\right)\right\rangle=\left\langle L_{n-1} X\right\rangle$ when $X$ is finite.

When $G=\Sigma_{p}$, the symmetric group on $p$ letters, this is related to a conjecture of Hopkins and Mahowald (usually framed in terms of Mahowald's functor $\left.\mathbf{R} P_{-\infty}(-)\right)$.
\end{abstract}

\section{INTRODUCTION}

We briefly recall the spectra that occur in Lin's proof of the Segal conjecture for the group $\mathbf{Z} /(2)$. Embed $\mathbf{Z} /(p)$ into $S^{1}$ and look at the pullback of the tautological (complex) line bundle over $B S^{1}$ as a bundle over $B \mathbf{Z} /(p)$. Call this bundle $\xi$.

We denote by $P_{-2 k}$ the spectrum given by the Thom spectrum $(B \mathbf{Z} /(p))^{-k \xi}$ when $p=2$, or when $p$ is odd the summand of that spectrum corresponding to $B \Sigma_{p}$. (We refer the reader to [20] for the definition of a Thom spectrum associated to a virtual bundle.) $P_{-2 k}$ has a cell in every dimension $\geq-2 k$ and is the spectrum frequently called $\mathbf{R} P_{-2 k}^{\infty}$ when $p=2$. When $p$ is odd, $P_{-2 k}$ has a cell in every dimension congruent to 0 or -1 modulo $q=2 p-2$ and $\geq-2 k$. $P_{-2 k}$ is the same as the spectrum denoted $P_{-2 k}^{\infty}$ in [19], and constructed there by James periodicity rather than via Thom spectra.

Lin's theorem [10] (Gunarwardena's theorem when $p>2$ [1]) states that

$$
\lim _{k}\left(P_{-2 k} \wedge X\right)=\Sigma^{-1} X_{p}^{\wedge}
$$

when $X$ is a finite spectrum. This inverse system of spectra (with some minor alterations) is also what is used to define the root invariant (see [11] for $p=2$, or more generally, $[13,19])$. As shorthand, we write $P_{-\infty}(X)$ for $\lim _{\longleftarrow}\left(P_{-2 k} \wedge X\right)$.

Mahowald and Ravenel [12] have conjectured a relationship between chromatic periodicity and Mahowald's root invariant. There is a related conjecture by Hopkins

Received by the editors January 19, 1995 and, in revised form, June 8, 1995.

1991 Mathematics Subject Classification. Primary 55P60, 55P42; Secondary 55N91.

The authors were partially supported by the NSF. 
and Mahowald that is more closely related to our concerns in this paper. Denote Bousfield localization with respect to $E(n)$ by $L_{n}$. They conjecture that

$$
P_{-\infty}\left(L_{n} X\right)=\Sigma^{-2} L_{n-1} X_{p}^{\wedge} \vee \Sigma^{-1} L_{n-1} X_{p}^{\hat{p}}
$$

for $X$ finite. (A word to the experts: this conjecture is connected with Hopkins's chromatic splitting conjecture (see [7]) and at the time (2) was conjectured the chromatic splitting conjecture was in too simple a form. In light of its current corrected form as in [7], (2) is probably also too optimistic, though we expect it is true as stated when $X$ has type $n-1$.)

Greenlees and May put the $P_{-\infty}$ construction in a more general context in [4]. There they define the Tate $G$-spectrum associated to a $G$-spectrum $X, t_{G}(X)$. We are not concerned with equivariant spectra here, but we use $t_{G}$ to construct a functor from (ordinary) spectra to spectra. We abuse notation and write $i_{*}$ for the functor which is the composite of the inclusion of ordinary spectra into the category of naive $G$-spectra (as the objects on which $G$ acts trivially) with the left adjoint of the forgetful functor from $G$-spectra to naive $G$-spectra. So $i_{*}$ is a functor from ordinary spectra to $G$-spectra. Our other functor is the $G$-fixed point functor, $(-)^{G}$, which goes from $G$-spectra to spectra. We refer the reader to [9] for details. We define

$$
P_{G}(X)=t_{G}\left(i_{*} X\right)^{G}
$$

Then $[4,16.1]$ shows that

$$
P_{\Sigma_{p}}(X)=P_{-\infty}(\Sigma X)
$$

(the left-hand side needs to be localized at $p$ when $p$ is odd).

Henceforth we will only be concerned with the case where $G$ is a finite group. We now have a family of functors, one for each finite group $G$, and we assume (to guarantee non-triviality of our functors) that $p$ divides the order of $G$. Our main theorem is the following.

Theorem 1.1. Let $X$ be a finite spectrum. Then $\left\langle P_{G}\left(L_{n} X\right)\right\rangle=\left\langle L_{n-1} X\right\rangle$.

Here $\langle X\rangle$ means the Bousfield class of the spectrum $X$ as given in [2].

We also give a result about complex oriented $v_{n}$-periodic spectra.

Theorem 1.2. If $E$ is Landweber exact and $v_{n}$-periodic, then $P_{\mathbf{Z} /(p)} E$ is Landweber exact and $v_{n-1}$-periodic. It follows generally that $\left\langle P_{G}\left(E_{(p)}\right)\right\rangle=\langle E(n-1)\rangle$.

Our proofs rely on $\left[5\right.$, Theorem 1.1], which implies that $P_{G}(K(n)) \simeq *$. We also use two other results that are relatively well known. We use Ravenel's Proposition 1.34 from [16]:

$$
\langle X\rangle=\langle C(f)\rangle \vee\langle\operatorname{Tel}(f)\rangle
$$

where $f$ is a self-map of $X, C(f)$ is the cofiber and $T e l(f)$ is the mapping telescope. Finally, we use a theorem of Hopkins and Ravenel from [18] to show that $P_{G}(K(n)) \simeq *$ implies $P_{G}\left(L_{n} X\right) \simeq *$ when $X$ is finite type $n$.

Using the interesting results of Mahowald and Shick in [15] one can show that $P_{\mathbf{Z} /(2)}(\operatorname{Tel}(X)) \simeq *$ where $X$ is finite type $n$ and $\operatorname{Tel}(X)$ is the mapping telescope of $X$ under a $v_{n}$ map. One can use this to deduce our theorems in the special case $G=\mathbf{Z} /(2)$. Chun-Nip Lee has done this independently [8].

The authors would like to thank Neil Strickland for pointing out various places where we failed to say what we meant or to mean what we said in a previous draft. 


\section{There is a Finite type $n$ SPectrum $F$ with $L_{n} F K(n)$-Nilpotent}

Recall that $X$ is said to be $E$-prenilpotent if $L_{E} X$ is $E$-nilpotent; that is if $L_{E} X$ can be built up in finitely many stages from spectra of the form $E \wedge Z$ by taking cofibrations and retracts. Note that $N$ is $E$-nilpotent implies $N \wedge M$ is $E \wedge M$-nilpotent. By $[18,8.3]$ there is a finite type 0 spectrum $Y$ that is $L_{n} B P$ prenilpotent.

By [18, Lemma 8.1.4]

$$
\left\langle L_{n} B P\right\rangle=\left\langle v_{n}^{-1} B P\right\rangle,
$$

which is in turn equal to $\langle E(n)\rangle$ by [16]. So $L_{n} Y$ is $L_{n} B P$-nilpotent. Now let $M$ be a finite type $n$ spectrum with

$$
B P_{*} M=B P_{*} /\left(p^{i_{0}}, \ldots, v_{n-1}^{i_{n-1}}\right)
$$

and such that $M$ is a ring spectrum. (See [3] for the existence of such ring spectra.) Then $B P \wedge M=B P /\left(p^{i_{0}}, \ldots, v_{n-1}^{i_{n-1}}\right)$, so by [17, Theorem 1],

$$
L_{n} B P \wedge M=v_{n}^{-1} B P \wedge M
$$

But $v_{n}^{-1} B P \wedge M$ is made out of finitely many cofibrations with cofiber $v_{n}^{-1} B P / I_{n}=$ $B(n)$. Now by [21, Remark 6.19], $B(n)=K(n) \wedge B$ for some $B$, so it follows that $L_{n} B P \wedge M$ is $K(n)$-nilpotent.

Since $L_{n} Y \wedge M$ is $L_{n} B P \wedge M$-nilpotent, it follows that $L_{n} Y \wedge M$ is $K(n)$-nilpotent.

Lemma 2.1. There is a finite type $n$ spectrum $F$ with $L_{n} F K(n)$-nilpotent.

Proof. Take $F=Y \wedge M$.

$$
\text { 3. } P_{G}\left(L_{n} X \wedge F\right) \simeq * \text { IF } F \text { IS TYPE } n
$$

We recall from [5] that $t_{G}\left(i_{*} K(n)\right) \simeq *$ as a $G$-spectrum. It follows that $P_{G}(K(n)) \simeq *$. We record the following lemma.

Lemma 3.1. If $X$ is $K(n)$-nilpotent, then $P_{G}(X) \simeq *$.

Proof. First note that since $P_{G}$ takes cofibrations to cofibrations, it suffices to prove that $P_{G}(K(n) \wedge Z) \simeq *$ for any $Z$. But $P_{G}(R)$ is a ring spectrum when $R$ is a ring spectrum, and $P_{G}(N)$ is a module spectrum over $P_{G}(R)$ if $N$ is a module spectrum over $R$ [4, Proposition 3.5]. It follows that $P_{G}(K(n) \wedge Z) \simeq *$.

Remark. The same proof shows that $t_{G}(X) \simeq *$ equivariantly if $X$ is $i_{*} K(n)$ nilpotent in the category of $G$-spectra.

Corollary 3.2. If $F$ is finite type $n$, then $P_{G}\left(L_{n} X \wedge F\right) \simeq *$ for any spectrum $X$.

Proof. Let $\mathcal{C}$ be the category of finite spectra $F$ such that $P_{G}\left(L_{n} X \wedge F\right) \simeq *$ for all spectra $X . \mathcal{C}$ is a thick subcategory in the sense of [6]. It follows that if $\mathcal{C} \cap \mathcal{C}_{n} \neq \emptyset$, then $\mathcal{C}_{n} \subseteq \mathcal{C}$. We recall that if a spectrum $Y$ is $K(n)$-nilpotent, so is $X \wedge Y$ for any spectrum $X$. Since $L_{n} X \wedge F=X \wedge L_{n} F$ ( $L_{n}$ is smashing) by Lemma 3.1 and Lemma $2.1, \mathcal{C} \cap \mathcal{C}_{n} \neq \emptyset$.

Remark. Since $L_{n} F=L_{K(n)} F$ when $F$ is finite type $n$, one might ask when $P_{G}\left(L_{K(n)} X\right) \simeq *$. While we don't know the most general answer, this does not hold in general for $X$ finite. Using the methods of this paper, one can easily check that if $X$ is finite, $\left\langle P_{G}\left(L_{K(n)} X\right)\right\rangle=\left\langle L_{n-1} X\right\rangle$. 
We use equation (3) inductively. We get

$$
\begin{aligned}
\left\langle P_{G} L_{n} X\right\rangle= & \left\langle p^{-1} P_{G} L_{n} X\right\rangle \vee\left\langle P_{G} L_{n} X \wedge M\left(p^{i_{0}}\right)\right\rangle \\
= & \left\langle p^{-1} P_{G} L_{n} X\right\rangle \vee\left\langle v_{1}^{-1} P_{G} L_{n} X \wedge M\left(p^{i_{0}}\right)\right\rangle \vee \cdots \\
& \vee\left\langle v_{n-1}^{-1} P_{G} L_{n} \wedge M\left(p^{i_{0}}, \ldots, v_{n-2}^{i_{n-2}}\right)\right\rangle \vee\left\langle P_{G} L_{n} X \wedge M\left(p^{i_{0}}, \ldots, v_{n-1}^{i_{n-1}}\right)\right\rangle .
\end{aligned}
$$

Now since the $P_{G} L_{n} X \wedge M\left(p^{i_{0}}, \ldots, v_{n-1}^{i_{n-1}}\right) \simeq *$ by Corollary 3.2, we get

$$
\begin{aligned}
\left\langle P_{G} L_{n} X\right\rangle= & \left\langle p^{-1} P_{G} L_{n} X\right\rangle \vee\left\langle v_{1}^{-1} P_{G} L_{n} X \wedge M\left(p^{i_{0}}\right)\right\rangle \vee \cdots \\
& \vee\left\langle v_{n-1}^{-1} P_{G} L_{n} \wedge M\left(p^{i_{0}}, \ldots, v_{n-2}^{i_{n-2}}\right)\right\rangle .
\end{aligned}
$$

Since $P_{G} L_{n} X$ is an $L_{n} S^{0}$ module, it follows that

$$
\begin{aligned}
v_{j}^{-1} P_{G} L_{n} X \wedge M\left(p^{i_{0}}, \ldots, v_{j-1}^{i_{j-1}}\right) & =P_{G} L_{n} X \wedge v_{j}^{-1} L_{n} M\left(p^{i_{0}}, \ldots, v_{j-1}^{i_{j-1}}\right) \\
& =\left(P_{G} L_{n} X\right) \wedge L_{j} M\left(p^{i_{0}}, \ldots, v_{j-1}^{i_{j-1}}\right) .
\end{aligned}
$$

(The last equality follows from $\left[14\right.$, Proposition 6.1].) Since $\left\langle L_{j} M\left(p^{i_{0}}, \ldots, v_{j-1}^{i_{j-1}}\right)\right\rangle=$ $\langle K(j)\rangle$, we see that

$$
\left\langle P_{G} L_{n} X\right\rangle \leq\langle K(0)\rangle \vee \cdots \vee\langle K(n-1)\rangle=\langle E(n-1)\rangle .
$$

Now since $L_{n} X$ is an $L_{n} S^{0}$ module, $P_{G} L_{n} X$ is a $P_{G} L_{n} S^{0}$ module [4, Proposition 3.5]. But $P_{G} L_{n} S^{0}$ is self-local since it is a ring spectrum [16], so by equation (4) $P_{G} L_{n} S^{0}$ is $E(n-1)$-local, hence so is $P_{G} L_{n} X$.

To finish the proof of Theorem 1.1 it remains to show the inequality in equation (4) is actually an equality when $X=S^{0}$. In section 6 we use Theorem 1.2 to do this.

\section{5. $P_{G}$ OF LANDWEBER EXACT $v_{n}$-PERIODIC THEORIES}

In this section we prove Theorem 1.2. We will suppose that $E$ is a complex oriented homology theory. We also assume that $E$ is $p$-local $\left(P_{G}(E)=P_{G}\left(E_{(p)}\right)\right.$ if $G$ happens to be a $p$-group). Then we can assume $E$ is oriented by a map from $B P$, so that we can consider $v_{i}$ as an element of $E_{*}$. We remind the reader that $I_{j}=\left(p, v_{1}, \ldots, v_{j-1}\right)$, and that for $B P$ (and hence for any spectrum oriented from $B P)$,

$$
[p](x)=p x+{ }_{F} v_{1} x^{p}+{ }_{F} v_{2} x^{p^{2}}+{ }_{F} \cdots+{ }_{F} v_{i} x^{p^{i}}+{ }_{F} \cdots
$$

where $+_{F}$ is the sum in the formal group law on $E_{*}$.

We begin by remarking that for complex oriented $E$ in which the leading coefficient of $[p](x)$ is not a 0 -divisor,

$$
\pi_{*} P_{\mathbf{Z} /(p)} E=E_{*}((x)) /([p](x))
$$

where $|x|=-2, E_{*}((x))$ denotes the ring of Laurent series over $E_{*}$ which have only finitely many terms involving negative powers of $x$, and $[p](x)$ is the $p$-series. It follows that when $[p](x)$ is not a zero divisor, we have a short exact sequence

$$
E_{*}((x)) \stackrel{\cdot[p](x)}{\longrightarrow} E_{*}((x)) \rightarrow \pi_{*} P_{\mathbf{Z} /(p)} E .
$$

We now assume that $E$ is $v_{n}$-periodic Landweber exact. We define $v_{n}$-periodic almost as in [5, Definition 1.3]; $E$ is $v_{n}$-periodic if $v_{n}$ is a unit on $E_{*} / I_{n}$ and in addition $E_{*} / I_{n} \neq 0$. 
For each $j \leq n$, we know that $E_{*} / I_{j} \rightarrow v_{j}^{-1} E_{*} / I_{j}$ is injective by the hypothesis of Landweber exactness. It follows that

$$
E_{*}((x)) / I_{j}=E_{*} / I_{j}((x)) \rightarrow\left(v_{j}^{-1} E_{*} / I_{j}\right)((x))
$$

is injective also. Now $[p](x)$ is a unit in $\left(v_{j}^{-1} E_{*} / I_{j}\right)((x))$ since it is a power series with leading term $v_{j} x^{p^{j}}$, which is a unit. It follows that

$$
E_{*}((x)) / I_{j} \rightarrow\left(v_{j}^{-1} E_{*} / I_{j}\right)((x)) \stackrel{\cdot[p](x)}{\longrightarrow}\left(v_{j}^{-1} E_{*} / I_{j}\right)((x))
$$

is injective, hence

$$
E_{*}((x)) / I_{j} \stackrel{\cdot[p](x)}{\longrightarrow} E_{*}((x)) / I_{j}
$$

is also.

We examine the diagram of short exact sequences below, in which the bottom row is the cokernel of the map between the top two rows.

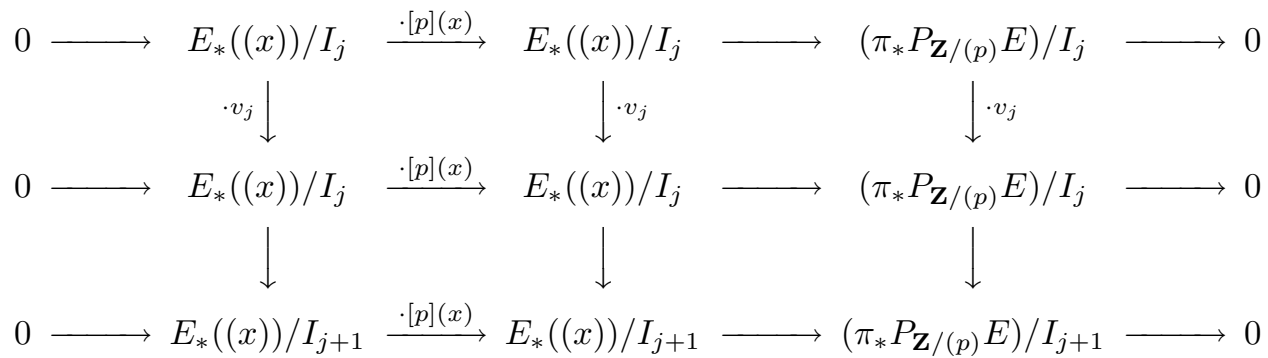

By the snake lemma applied to the first two rows (together with the observation that the first two vertical maps are injective) we see that $v_{j}$ is injective on $\left(\pi_{*} P_{\mathbf{Z} /(p)} E\right) / I_{j}$.

We can also see that $[p](x)$ is not a unit in $E_{*}((x)) / I_{j}$ unless $v_{j}$ is a unit in $E_{*} / I_{j}$, therefore $\left(\pi_{*} P_{\mathbf{Z} /(p)} E\right) / I_{j} \neq 0$ unless $j=n$, and this last observation tells us that $v_{n-1}$ is a unit on $\left(\pi_{*} P_{\mathbf{Z} /(p)} E\right) / I_{n-1}$.

We conclude that $P_{\mathbf{Z} /(p)} E$ is Landweber exact, and that

$$
\pi_{*}\left(P_{\mathbf{Z} /(p)} E\right) / I_{n-1} \neq 0
$$

while $\pi_{*}\left(P_{\mathbf{Z} /(p)} E\right) / I_{n}=0$. It follows by using (3) as before (see [7, Corollary 1.12]) that

$$
\left\langle P_{\mathbf{Z} /(p)} E\right\rangle=\langle E(n-1)\rangle .
$$

By using the maps of complex oriented ring spectra

$$
E \rightarrow P_{G} E \rightarrow P_{\mathbf{Z} /(p)} E
$$

(when $\mathbf{Z} /(p) \subseteq G$ ) we also deduce that

$$
\left\langle P_{G} E\right\rangle=\langle E(n-1)\rangle .
$$

\section{Proof of Theorem 1.1}

We recall from section 4 that $v_{j}^{-1} P_{G} L_{n} S^{0} \wedge M\left(p^{i_{0}}, \ldots, v_{j-1}^{i_{j-1}}\right)$ has the Bousfield class of either a point or of $K(j)$. So to show it has the class of $K(j)$, we need only show that it is not contractible. 
We pick $i_{0}, \ldots, i_{j-1}$ so that $M\left(p^{i_{0}}, \ldots, v_{j-1}^{i_{j-1}}\right)$ is a ring spectrum. Then we observe that the map

$$
\begin{gathered}
S^{0} \rightarrow v_{j}^{-1} P_{G} L_{n} S^{0} \wedge M\left(p^{i_{0}}, \ldots, v_{j-1}^{i_{j-1}}\right) \rightarrow v_{j}^{-1} P_{\mathbf{Z} /(p)} L_{n} S^{0} \wedge M\left(p^{i_{0}}, \ldots, v_{j-1}^{i_{j-1}}\right) \rightarrow \\
v_{j}^{-1} P_{\mathbf{Z} /(p)} E(n) \wedge M\left(p^{i_{0}}, \ldots, v_{j-1}^{i_{j-1}}\right) \stackrel{=}{\longrightarrow} v_{j}^{-1} P_{\mathbf{Z} /(p)} E(n) /\left(p^{i_{0}}, \ldots, v_{j-1}^{i_{j-1}}\right) \rightarrow \\
v_{j}^{-1} P_{\mathbf{Z} /(p)} E(n) /\left(p, \ldots, v_{j-1}\right)
\end{gathered}
$$

is the unit of the ring spectrum $v_{j}^{-1} P_{\mathbf{Z} /(p)} E(n) /\left(p, \ldots, v_{j-1}\right)$. This is non-zero if $j<n$ by Theorem 1.2. So none of the intervening spectra are contractible either.

For arbitrary finite $X$ (instead of $\left.S^{0}\right)$ just smash with $X$. Note that $\langle-\rangle, P_{G}(-)$, and localization commute with smashing with a finite spectrum.

Remark. The same proof can be iterated to draw the obvious conclusions about $P_{G}^{k}\left(L_{n} S^{0}\right)$.

\section{REFERENCES}

1. J. F. Adams, J. H. Gunarwardena, and H. R. Miller, The Segal conjecture for elementary abelian p-groups, Topology, 24 (1985), 435-460. MR 87m:55026

2. A. K. Bousfield, The localization of spectra with respect to homology, Topology, 18 (1979), 257-281. MR 80m:55006

3. E. S. Devinatz, Small ring spectra, Journal of Pure and Applied Algebra 81 (1992), 11-16. MR 93g:55012

4. J. P. C. Greenlees and J. P. May, Generalized Tate cohomology, volume 543, Amer. Math. Soc., Providence, Rhode Island, 1995. CMP 95:07

5. J. P. C. Greenlees and H. Sadofsky, The Tate spectrum of $v_{n}$-periodic complex oriented theories, submitted.

6. M. J. Hopkins and J. H. Smith, Nilpotence and stable homotopy theory II (to appear).

7. M. Hovey, Bousfield localization functors and Hopkins' chromatic splitting conjecture, In M. Cenkl and H. Miller, editors, The Čech Centennial, 1993, volume 181 of Contemporary Mathematics, pages 225-250, Providence, Rhode Island, 1995. American Mathematical Society.

8. C.-N. Lee, Bousfield equivalence classes and the Lin tower, preprint, 1994.

9. L. G. Lewis, J. P. May, and M. Steinberger, Equivariant Stable Homotopy Theory, Lecture Notes in Mathematics, vol. 1213, Springer-Verlag, New York, 1986. MR 88e:55002

10. W. H. Lin, On conjectures of Mahowald, Segal and Sullivan, Proc. Cambridge Phil. Soc. 87 (1980), 449-458. MR 81e:55020

11. M. E. Mahowald, The Metastable Homotopy of $S^{n}$, Memoirs of the Amer. Math. Soc., vol. 72, American Mathematical Society, Providence, Rhode Island, 1967. MR 38:5216

12. M. E. Mahowald and D. C. Ravenel, Toward a global understanding of the homotopy groups of spheres, In Samuel Gitler, editor, The Lefschetz Centennial Conference: Proceedings on Algebraic Topology, volume 58 II of Contemporary Mathematics, pages 57-74, Providence, Rhode Island, 1987. American Mathematical Society. MR 88k:55011

13. M. E. Mahowald and D. C. Ravenel, The root invariant in homotopy theory, Topology 32 (1993), 865-898. MR 94h:55025

14. M. E. Mahowald and H. Sadofsky, $v_{n}$-telescopes and the Adams spectral sequence, Duke Math. J. 78 (1995), 101-129.

15. M. E. Mahowald and P. Shick, Root invariants and periodicity in stable homotopy theory, Bull. London Math. Soc. 20 (1988), 262-266. MR 89b:55009

16. D. C. Ravenel, Localization with respect to certain periodic homology theories, American Journal of Mathematics 106 (1984), 351-414. MR 85k:55009

17. D. C. Ravenel, The geometric realization of the chromatic resolution, In W. Browder, editor, Algebraic topology and algebraic K-theory, pages 168-179, 1987. MR 89d:55050 
18. D. C. Ravenel, Nilpotence and Periodicity in Stable Homotopy Theory, Ann. of Math. Stud., vol. 128, Princeton University Press, Princeton, 1992. MR 94b:55015

19. H. Sadofsky, The root invariant and v1-periodic families, Topology 31 (1992), 65-111. MR 92k:55021

20. R. Switzer, Algebraic Topology - Homotopy and Homology, Springer-Verlag, New York, 1975. MR 52:6695

21. U. Würgler, Cobordism theories of unitary manifolds with singularities and formal group laws, Math. Z. 150 (1976), 239-260. MR 54:6173

Department of Mathematics, Massachusetts Institute of Technology, Cambridge, MASSACHUSETTS 02139

E-mail address: hovey@math.mit.edu

Department of Mathematics, Johns Hopkins University, Baltimore, Maryland 21230

E-mail address: hs@math.jhu.edu

Current address: Department of Mathematics, University of Oregon, Eugene, Oregon 97403

E-mail address: sadofsky@math.uoregon.edu 